\title{
Emergent Statistical Mechanics from Properties of Disordered Random Matrix Product States
}

\author{
Jonas Haferkamp, ${ }^{1,2,{ }^{*}}$ Christian Bertoni $\odot,{ }^{1}$ Ingo Roth $\odot,{ }^{1,3}$ and Jens Eisert ${ }^{1,2}$ \\ ${ }^{1}$ Dahlem Center for Complex Quantum Systems, Freie Universität Berlin, Berlin 14195, Germany \\ ${ }^{2}$ Helmholtz-Zentrum Berlin für Materialien und Energie, Berlin 14109, Germany \\ ${ }^{3}$ Quantum Research Centre, Technology Innovation Institute (TII), Abu Dhabi
}

(Received 16 April 2021; revised 13 July 2021; accepted 24 August 2021; published 13 October 2021)

\begin{abstract}
The study of generic properties of quantum states has led to an abundance of insightful results. A meaningful set of states that can be efficiently prepared in experiments are ground states of gapped local Hamiltonians, which are well approximated by matrix product states. In this work, we introduce a picture of generic states within the trivial phase of matter with respect to their nonequilibrium and entropic properties. We do so by rigorously exploring nontranslation-invariant matrix product states drawn from a local independent and identically distributed Haar measure. We arrive at these results by exploiting techniques for computing moments of random unitary matrices and by exploiting a mapping to partition functions of classical statistical models, a method that has lead to valuable insights on local random quantum circuits. Specifically, we prove that such disordered random matrix product states equilibrate exponentially well with overwhelming probability under the time evolution of Hamiltonians featuring a nondegenerate spectrum. Moreover, we prove two results about the entanglement Rényi entropy: the entropy with respect to sufficiently disconnected subsystems is generically extensive in the system size, and for small connected systems, the entropy is almost maximal for sufficiently large bond dimensions.
\end{abstract}

DOI: 10.1103/PRXQuantum.2.040308

The application of random matrix theory to the study of interacting quantum many-body systems has proven to be a particularly fruitful endeavor, in fact, in various readings. This includes countless applications in condensed matter physics, allowing us to predict a wide range of topics ranging from universal conductance fluctuations, weak localization, or coherent backscattering [1]. In a mindset closer to notions of quantum information theory, it has been shown that, for uniformly drawn states, such ideas lead to a principle of maximum entropy [2-4], an insight that has implications in the context of quantum computing [5]. That said, from an operational perspective, quantum states that are uniformly random - in the sense that they are drawn from a global invariant measure - are not particularly natural in many contexts. From a physical perspective, such random states do not respect locality present in most naturally occurring systems. What is more common and natural, in contrast, are quantum states that emerge from ground states of gapped Hamiltonians. Indeed, in common experiments, often good approximations of ground

\footnotetext{
*jonas.haferkamp@fu-berlin.de
}

Published by the American Physical Society under the terms of the Creative Commons Attribution 4.0 International license. Further distribution of this work must maintain attribution to the author(s) and the published article's title, journal citation, and DOI. states of local Hamiltonian characterized by finite-ranged interactions can be feasibly prepared, by means of cooling procedures or by resorting to suitable loading procedures. A key question that arises, therefore, is to what extent one can expect such ground states to exhibit the same or similar properties as (Haar-random) uniformly chosen ones.

This question can be interpreted in several readings. A particularly important one from the perspective of outof-equilibrium physics is, specifically, to what extent such states would eventually equilibrate in time under the evolution of general Hamiltonians [6-8]. Equilibration is an important concept in the foundations of quantum statistical mechanics and considerations of how apparent equilibrium states seem to emerge under closed system quantum dynamics. Equilibration refers to properties becoming apparently and effectively stationary, even though the entire system would remain to undergo unitary dynamics generated by some local Hamiltonian.

In this work, we consider such typical ground states from a fresh perspective. More precisely, we prove several concentration-type results for so-called matrix product states (MPSs), instances of tensor network states that approximate ground states of gapped one-dimensional quantum systems well. This class of states hence indeed captures those that can be obtained by cooling local Hamiltonians with a spectral gap. To pick such random states seems a most meaningful approach to respect natural restrictions of locality. Since one can readily see such 
states as being ground states of disordered parent Hamiltonians [9], they can be viewed as being typical representatives of one-dimensional quantum phases of matter. Indeed, while the significance of random ensembles of quantum states respecting locality has been appreciated early on [10], only recently, first steps have been taken towards a rigorous understanding of such ensembles, specifically concerning spectral properties and decays of correlations of generic MPSs [11,12].

Specifically, the powerful technical tool we bring in to this kind of study is a framework - seemingly out of context - related to mappings to partition functions of a one-dimensional statistical mechanical models. We also develop this picture further. Equipped with this technical tool, we prove with overwhelming probability that a randomly chosen MPS - drawn according to the independent and identically distributed (i.i.d.) Haar measure in the physical and the virtual dimensions of the tensor network state - equilibrates exponentially under the time evolution of Hamiltonians with nondegenerate spectrum. Moreover, as a second result, and also motivated by the equilibration of systems exhibiting many-body localization [13-15], we prove the extensivity of the Rényi-2 entropy with overwhelming probability with respect to equidistant disconnected subsystems. This result complements recent work obtained for translation-invariant MPSs [16]. A third result is an improved principle of maximum entropy for disordered random MPSs. More precisely, we show that the Rényi-2 entropy is almost maximal for small connected subsystems up to errors polynomially small in the bond dimension $D$. This again complements results for random translation-invariant MPSs [11,17]. Another complementary result is given in Refs. $[18,19]$, where formulas for the asymptotic values of various quantum entropies are derived in the setting of ergodic Markov chains.

Again, this substantial progress in studying generic equilibrium and out-of-equilibrium states of matter are facilitated by a technical tool introduced to the context at hand. The proofs of the above results are based on a graphical calculus, as all of them are obtained via mappings to partition functions of a one-dimensional statistical mechanical model. These models can be obtained by an application of the Weingarten calculus. In fact, the method we make use of is inspired by recent work on random quantum circuits. Such random quantum circuits have recently prominently been discussed in the literature, both in the context of quantum computing where they are used to show a quantum advantage over classical algorithms [20,21], as well as in proxies for scrambling dynamics [22-29]. In that context, similar mappings have been exploited [26]. We showcase here, therefore, how this mapping can be a very much helpful tool in a conceptually very different context. We also make use of a generalization of the Cauchy-Schwarz inequality to tensor networks without self-contractions [30]. In the Appendix, we argue that the low-entanglement structure of MPSs prevents them from having generic properties of uniform states in the sense that they will now form an approximate complex projective 2-design [31] in a meaningful sense. The upshot of this work is that, using a machinery of mappings to partition functions of a one-dimensional statistical mechanical model and the Weingarten calculus, a wealth of out-of-equilibrium and equilibrium properties of generic one-dimensional quantum phases of matter can be rigorously computed.

\section{SETTING}

\section{A. Random matrix product states}

We start by stating the underlying model of random quantum states used throughout this work. A very reasonable model of random matrix product states already used in Ref. [10] is the following. Consider an arbitrary MPS vector [32,33] with periodic boundary conditions and $n$ constituents of local dimension $d$. Such a state vector can be written as

$$
|\psi\rangle=\sum_{i_{1}, \ldots, i_{n}} \operatorname{Tr}\left[A_{i_{1}}^{(1)} A_{i_{2}}^{(2)} \cdots A_{i_{n}}^{(n)}\right]\left|i_{1}, \ldots, i_{n}\right\rangle
$$

Here, the $A^{(i)}$ are complex valued $D \times D$ matrices that specify the quantum state at hand, where $D$ is referred to as the bond dimension, sometimes also called the tensor train rank. In a commonly used graphical calculus, this is represented as

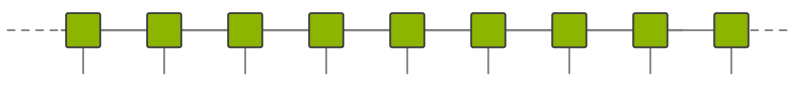

We consider MPSs that can be unitarily embedded [34,35]. Here, one equips each tensor with another leg and feeds in an arbitrary state vector $|0\rangle \in \mathbb{C}^{d}$ to get

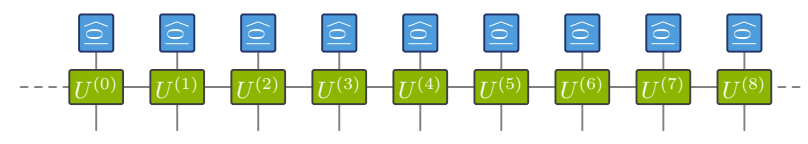

Each unitary $U^{(0)}, \ldots, U^{(n)} \in U(d D)$ can be seen as mapping one $\mathbb{C}^{d} \otimes \mathbb{C}^{D}$ input system to another $\mathbb{C}^{d} \otimes \mathbb{C}^{D}$ output system. These can be normalized by choosing an appropriate normalization for the boundary vectors. We can construct MPSs with periodic boundary conditions analogously. This motivates a very natural probability measure.

Definition 1 (Random matrix product state). A random matrix product state (RMPS) of local dimension d, system size $n$, and bond dimension D is a state defined by Eq. (3) 
with each unitary $U^{(1)}, \ldots, U^{(n)} \in U(d D)$ drawn i.i.d. randomly from the Haar measure. We denote the resulting measure as $\mu_{d, n, D}$.

Note that this definition can be regarded as drawing the $A^{(i)}$ tensor cores uniformly from the Stiefel manifold of isometries. This probability measure makes a lot of sense: it is a distribution over random disordered, nontranslationinvariant quantum states. Once again, each realization will have a parent Hamiltonian [9], a gapped local Hamiltonian for which the given state is an exact ground state. In this sense, the probability measure discussed here can equally be seen as a probability measure on disordered, random local Hamiltonians. It should be noted that this probability measure takes values on state vectors that are not exactly normalized. It is, however, easy to see that the values of $\langle\psi \mid \psi\rangle$ are strongly concentrated around unity.

Lemma 1 (Concentration around a unit norm). It holds that

$$
\operatorname{Pr}(|\langle\psi \mid \psi\rangle-1| \geq \varepsilon) \leq \varepsilon^{-2} d^{-n} .
$$

This will be proven in Sec. V.

\section{B. Effective dimension and equilibration}

We now turn to discussing concepts of equilibration in quantum many-body dynamics. Equilibration refers to the observation that, for an observable $A$, the expectation value of a time-evolving quantum many-body system will for the overwhelming times take the same values as the expectation value with respect to the infinite time average: the expectation value then looks "equilibrated" [7,36]. For a given arbitrary state vector $|\psi\rangle$ that reflects the initial pure state at time $T=0$, this expectation value of an observable $A$ with respect to the infinite time average takes the form

$$
A_{\psi}^{\infty}:=\lim _{t \rightarrow \infty} \frac{1}{t} \int_{0}^{t}\left\langle\psi\left|A\left(t^{\prime}\right)\right| \psi\right\rangle d t^{\prime}
$$

The fluctuations around this infinite time average are defined as [36]

$$
\Delta A_{\psi}^{\infty}:=\lim _{t \rightarrow \infty} \frac{1}{t} \int_{0}^{t}\left|\left\langle\psi\left|A\left(t^{\prime}\right)\right| \psi\right\rangle-A_{\psi}^{\infty}\right|^{2} d t^{\prime} .
$$

The reduced density matrices on a local region are determined by local observables. It hence suffices to consider the above definitions. In particular, if $\Delta A_{\psi}^{\infty} \ll 1$ then the reduced density matrices on a small region cannot deviate from the infinite time average except for very brief time periods. In this sense a small value for $\Delta A_{\psi}^{\infty}$ implies equilibration.

\section{EQUILIBRATION OF RANDOM MATRIX PRODUCT STATES}

Equipped with these preparations, we are in the position to state our first main result. On the equilibration of RMPSs, following nonequilibrium dynamics, we prove the following theorem.

Theorem 1 (Equilibration of RMPSs). Let $H$ be a Hamiltonian with nondegenerate spectrum and nondegenerate spectral gap, and let $A$ be an observable evolving in time governed by $H$. Let $|\psi\rangle$ be a RMPS drawn from $\mu_{d, n, D}$ and $\left|\psi^{\prime}\right\rangle:=|\psi\rangle / \sqrt{\langle\psi \mid \psi\rangle}$. Then there are constants independent of the system parameters $c_{1}, c_{2}$ such that, for sufficiently large $n$, the infinite time average $\Delta A_{\psi^{\prime}}^{\infty}, E q$. (6), fulfills

$$
\operatorname{Pr}\left(\Delta A_{\psi^{\prime}}^{\infty} \leq e^{\left.-c_{1} \alpha(d, D) n\right)}\right) \geq 1-e^{-c_{2} \alpha(d, D) n}
$$

with

$$
\alpha(d, D)=\log \left(\frac{d-1 /\left(d D^{2}\right)}{(1+1 / D)[1+1 /(d D)]}\right) .
$$

This shows that, under the time evolution of many Hamiltonians, almost all matrix product states equilibrate exponentially well. The proof of Theorem 1 implies a second result that we can informally state as follows. Assume that we draw Hamiltonians from an ensemble such that all marginal distributions for most eigenstates are distributed according to the RMPS measure introduced above. Then, for an arbitrary initial state, the system equilibrates exponentially well with overwhelming probability. This is motivated by the fact that the equilibration of systems exhibiting many-body localization still lacks a completely rigorous explanation. In particular, it is known that the energy eigenstates of many-body localized systems satisfy an area law for the entanglement entropy $[14,37,38]$ and can be described by matrix product states [14]. It seems unrealistic that any natural ensemble of Hamiltonians has RMPSs as marginal eigenstates, but we believe that enough of this result might survive for more structured ensembles to prove equilibration of many-body localization systems.

\section{A. Proof techniques}

In this section, we present many of the proof techniques relevant for this work. In order to obtain Theorem 1, we observe that Theorem 3 of Ref. [39] can be generalized to any distribution $v$ on states provided that the effective dimension is large. The effective dimension measures how much overlap the initial state has with the energy eigenstates of the Hamiltonian. In particular, we have the following key result from Refs. [39-41] that we make use of. 
Lemma 2 ([39-41]). Consider a Hamiltonian with nondegenerate spectrum and nondegenerate spectral gaps, i.e., $E_{n}-E_{m}=E_{j}-E_{k}$ if and only if $n=j, m=k$, where $E_{n}$ labels the eigenvalues of the Hamiltonian. Then,

$$
\Delta A_{\psi}^{\infty}=O\left(1 / D_{e f f}\right)
$$

with

$$
1 / D_{e f f}:=\sum_{j}|\langle\psi \mid j\rangle|^{4}
$$

where $\{|j\rangle\}$ is the eigenbasis of the Hamiltonian $H$.

Theorem 1 follows immediately from Lemma 2 in combination with the following statement.

Lemma 3 (Bound to effective dimension). For all state vectors $|\phi\rangle$, we have

$$
\mathbb{E}_{\psi \sim \mu_{d, n, D}}|\langle\psi \mid \phi\rangle|^{4} \leq 2 \frac{(1+1 / D)^{n}[1+1 /(d D)]^{n}}{\left(d^{2}-1 / D^{2}\right)^{n}} .
$$

We are trying to find an upper bound on

$$
\mathbb{E}|\langle\psi \mid \phi\rangle|^{4}=\left\langle\left.\phi\right|^{\otimes 2} \mathbb{E}(|\psi\rangle\langle\psi|)^{\otimes 2} \mid \phi\right\rangle^{\otimes 2} .
$$

We make use of the Weingarten calculus [42,43], as elaborated upon here in the following statement.

Lemma 4 (Weingarten calculus). The th moment operator of Haar-random unitaries is given by

$$
\mathbb{E}_{U \sim \mu_{H}} U^{\otimes t} \otimes \bar{U}^{\otimes t}=\sum_{\sigma, \pi \in S_{t}} \operatorname{Wg}\left(\sigma^{-1} \pi, q\right)|\sigma\rangle\langle\pi|,
$$

where $|\sigma\rangle:=(\mathbb{1} \otimes r(\sigma))|\Omega\rangle$ with $r$ being the representation of the symmetric group $S_{t}$ on $\left(\mathbb{C}^{q}\right)^{\otimes t}$ that permutes the vectors in the tensor product, and $|\Omega\rangle=\sum_{j=1}^{q^{t}}|j, j\rangle$ the maximally entangled state vector up to normalization.

The Weingarten calculus is a powerful tool in particular when suitably combined with Penrose tensor-network diagrams providing a graphical calculus; e.g., for $t=2$ and $U(q)$, Eq. (13) takes the form

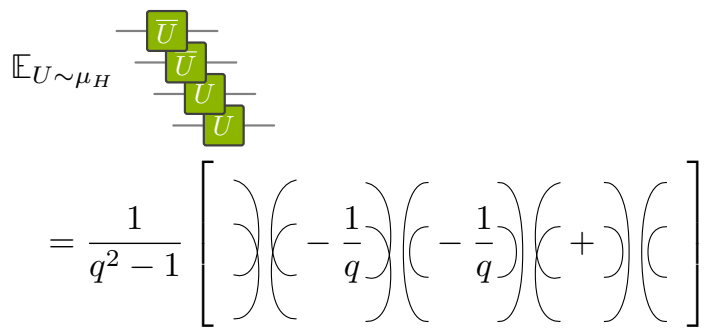

Graphically, we can express Eq. (12) as

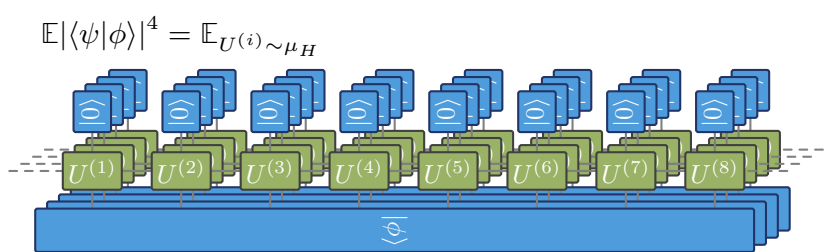

By evaluating each $\mathbb{E}_{U^{(i)}} U^{(i)} \otimes U^{(i)} \otimes \bar{U}^{(i)} \otimes \bar{U}^{(i)}$ individually according to Eq. (14), this can be reformulated as a partition function. Introducing the notation

$$
|\psi\rangle^{\otimes 2,2}:=|\psi\rangle^{\otimes 2} \otimes \overline{|\psi\rangle}^{\otimes 2},
$$

we obtain

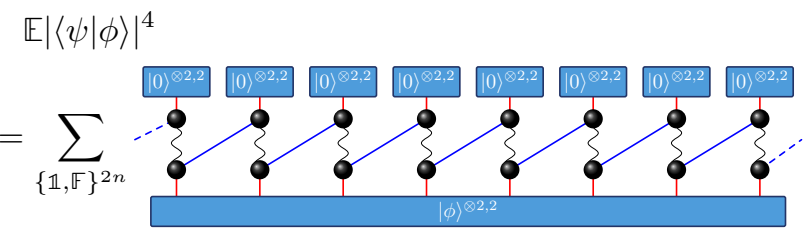

Here, the black balls correspond to a choice of an element of $S_{2}=\{\mathbb{1}, \mathbb{F}\}$ with $\mathbb{F}$ the swap permutation. The wiggly line corresponds to different weights for every pair of permutations $(\pi, \sigma)$ with the corresponding value of the Weingarten function $\mathrm{Wg}\left(\pi^{-1} \sigma, q\right)$ according to Eq. (14) with $q=d D$. The red edges denote contractions over $\mathbb{C}^{d}$ and the blue edges are contractions over $\mathbb{C}^{D}$. This is reminiscent of Ref. [26], where the frame potential of random quantum circuits is mapped to a partition function with local degrees of freedom corresponding to permutations. Note however that, for every permutation $\pi \in S_{2}$, we always have

$$
\langle\pi \mid 0\rangle^{\otimes 2,2}=|\langle 0 \mid 0\rangle|^{2}=1 .
$$

Moreover, every summand contains a factor of the form $\left\langle\left.\phi\right|^{\otimes 2,2} \bigotimes_{l=1}^{n} \mid \sigma_{l}\right\rangle$. We can bound this contribution using the following generalization of the Cauchy-Schwarz inequality [30].

Lemma 5 (Cauchy-Schwarz inequality for tensor networks [30]). Consider a tensor network $(T, C)$ with $J \geq 2$ tensors $T=\left(t^{j}\right)_{\in\{1, \ldots, J\}}$ such that no tensor in the contraction $C$ self-contracts, i.e., no string connects a tensor with itself. Then,

$$
|C(T)| \leq \prod_{j=1}^{J}\left\|t^{j}\right\|_{F},
$$

where $\|.\|_{F}$ is the Frobenius norm of the tensor $t^{j}$ viewed as a vector. 
Proof of Lemma 3. As the tensor network contraction $\left\langle\left.\phi\right|^{\otimes 2,2} \bigotimes_{l=1}^{n} \mid \sigma_{l}\right\rangle$ does not contain self-contractions, this yields

$$
\left.\left|\left\langle\left.\phi\right|^{\otimes 2,2} \bigotimes_{l=1}^{n} \mid \sigma_{l}\right\rangle\right| \leq \||| \phi\right\rangle\left.\right|_{F} ^{4}=1 .
$$

Therefore, we can apply a triangle inequality to the sum in Eq. (17) to obtain the bound

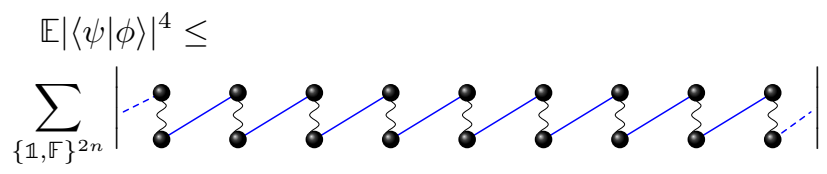

As contractions over $\mathbb{C}^{D}$ we have $\langle\mathbb{1} \mid \mathbb{1}\rangle=\langle\mathbb{F} \mid \mathbb{F}\rangle=D^{2}$ and

$$
\langle\mathbb{1} \mid \mathbb{F}\rangle=\langle\mathbb{F} \mid \mathbb{1}\rangle=D
$$

This allows us to obtain a sufficient upper bound on $1 / D_{\text {eff }}$ via a combinatorial argument. Consider a sequence $\left(\sigma_{1} \pi_{1}\right),\left(\sigma_{2} \pi_{2}\right), \ldots,\left(\sigma_{n} \pi_{n}\right) \in\{\mathbb{1}, \mathbb{F}\}^{2 n}$. Here, the $\sigma$ refer to the balls on top in Eq. (21) and $\pi$ to the ones on the bottom. If $\sigma_{i}=\pi_{i}$, the total interaction between sites $i$ and $i+1$ contributes with a term

$$
\frac{D^{2}}{d^{2} D^{2}-1}
$$

If $\sigma_{i} \neq \pi_{i}$, this contribution is divided by $d D$, and if $\pi_{i} \neq$ $\sigma_{i+1}$, it is divided by $D$. Hence, we sum over all possible ways of choosing $\sigma_{i} \neq \pi_{i}$ or $\pi_{i} \neq \sigma_{i+1}$, and divide by the corresponding factor, to get

$$
\begin{aligned}
\mathbb{E}|\langle\phi \mid \psi\rangle|^{4} & \leq 2 \frac{D^{2 n}}{\left(d^{2} D^{2}-1\right)^{n}} \sum_{l=0}^{n} \sum_{r=0}^{n}\left(\begin{array}{l}
n \\
l
\end{array}\right)\left(\begin{array}{l}
n \\
r
\end{array}\right) D^{-l-r} d^{-r} \\
& =2 \frac{(1+1 / D)^{n}[1+1 /(d D)]^{n}}{\left(d^{2}-1 / D^{2}\right)^{n}} .
\end{aligned}
$$

This implies Lemma 3.

Theorem 1 now follows from applying Lemma 3 to Eq. (10) together with an application of Markov's inequality and Lemma 1.
Proof of Theorem 1. By Lemma 3 we have

$$
\begin{aligned}
\mathbb{E}\left(1 / D_{\text {eff }}\right) & =\sum_{j} \mathbb{E}\left(|\langle\psi \mid j\rangle|^{4}\right) \\
& \leq 2 \frac{(1+1 / D)^{n}[1+1 /(d D)]^{n}}{\left[d-1 /\left(d D^{2}\right)\right]^{n}} \\
& =2 e^{-\alpha n}
\end{aligned}
$$

with $\alpha=\alpha(d, D)$ as defined in Eq. (8). Picking two positive constants $k_{1}, k_{2}>0$ with $k_{1}<1 / 2$, since $\Delta A_{\psi}^{\infty}=$ $O\left(1 / D_{\text {eff }}\right)$, Markov's inequality yields

$$
\operatorname{Pr}\left(\Delta A_{\psi}^{\infty} \leq e^{-k_{1} \alpha n}\right) \geq 1-e^{-k_{2} \alpha n} .
$$

Let $N:=\langle\psi \mid \psi\rangle$, and let $\left|\psi^{\prime}\right\rangle=N^{-1 / 2}|\psi\rangle$ be the normalized state vector. We then have

$$
\Delta A_{\psi^{\prime}}^{\infty}=\frac{\Delta A_{\psi}^{\infty}}{N^{2}}
$$

Suppose that $\Delta A_{\psi}^{\infty} \leq e^{-k_{1} \alpha n}$ and $|N-1| \leq e^{-k_{1} \alpha n}$. Then $N^{2} \geq\left(1-e^{-k_{1} \alpha n}\right)^{2}$ and

$$
\Delta A_{\psi^{\prime}}^{\infty} \leq \frac{e^{-k_{1} \alpha n}}{\left(1-e^{-k_{1} \alpha n}\right)^{2}} \leq e^{-c_{1} \alpha n}
$$

for some constant $c_{1}>0$ and the integer $n$ being large enough. Then, by the union bound, we get

$$
\begin{aligned}
\operatorname{Pr} & \left.\Delta A_{\psi^{\prime}}^{\infty} \leq e^{-c_{1} \alpha n}\right) \\
& \geq 1-\operatorname{Pr}\left(\Delta A_{\psi}^{\infty} \geq e^{-k_{1} \alpha n} \text { or }|N-1| \geq e^{-k_{1} \alpha n}\right) \\
& \geq \operatorname{Pr}\left(\Delta A_{\psi}^{\infty} \leq e^{-k_{1} \alpha n}\right)-\operatorname{Pr}\left(|N-1| \geq e^{-k_{1} \alpha n}\right) \\
& \geq 1-e^{-k_{2} \alpha n}-\operatorname{Pr}\left(|N-1| \geq e^{-k_{1} \alpha n}\right) \\
& \geq 1-e^{-k_{2} \alpha n}-e^{-2 k_{1} \alpha_{n}} d^{-n} \\
& \geq 1-e^{-k_{2} \alpha n}-e^{-\left(1-2 k_{1}\right) \alpha n}
\end{aligned}
$$

where we have used Lemma 1 and $\alpha \leq \log d$. Finally,

$$
\begin{aligned}
\operatorname{Pr}\left(\Delta A_{\psi^{\prime}}^{\infty} \leq e^{-c_{1} \alpha n}\right) & \geq 1-e^{-k_{2} \alpha n}-e^{-\alpha\left(1-2 k_{1}\right) n} \\
& \geq 1-e^{-c_{2} \alpha n}
\end{aligned}
$$

for a constant $c_{2}>0$ and sufficiently large $n$.

\section{EXTENSIVITY OF THE RÉNYI-2 ENTANGLEMENT ENTROPY}

In this section, we turn to proving our second result. This is once again motivated by the phenomenon of equilibration in quantum many-body physics and the endeavor to provide a rigorous foundation for it. It has been proven 
in Ref. [44] that systems equilibrate if their energy eigenstates have Rényi entropy that is extensive in the system size $n$, which means that

$$
S_{2}\left(\operatorname{Tr}_{A}[|j\rangle\langle j|]\right) \geq g(j) n
$$

for a sufficiently well-behaved function $g$ and some subsystem $A$. This property has been dubbed entanglement ergodicity [44]. Motivated by this insight, it has been proven in Ref. [16] that generic translation-invariant MPSs have extensive Rényi entropy if one considers a bipartition of the chain into the subsystem that corresponds to every $k$ th site and the rest. That is, the entropy grows proportional to the boundary $|\partial A|$. Considering this particular partitioning is enough, since one partition of the system satisfying Eq. (31) suffices to consider the system entanglement ergodic, and to show the equilibration properties proven in Ref. [44]. Here, we prove such a result with explicit quantitative bounds for disordered RMPSs with overwhelming probability. Interestingly, the details of the correlation length of the state is of no concern for the bound presented. Even though the correlation length is expected to be small compared to the system size but nonzero (compare also the results of Ref. [12] in the translationally invariant case), we still arrive at an extensive bound of the respective entropy, independent of how the distance $k$ between the sites precisely relates to the correlation length.

Theorem 2 (Extensivity of entanglement entropies). Suppose that $n$ is divisible by a positive integer $k$ and that $A$ consists of what remains after tracing out every $k$ th qudit. Let $\rho_{A}^{\prime}$ be the normalized density matrix of a RMPS drawn from $\mu_{d, n, D}$ reduced on $A$. Then,

$$
\operatorname{Pr}\left[S_{2}\left(\rho_{A}^{\prime}\right) \geq \Omega\left(\frac{n}{k}\right)\right] \geq 1-e^{-\Omega(n / k)} .
$$

Proof. The proof of Theorem 2 follows similar lines as that of Lemma 3. We first show that the purity $\left(\operatorname{Tr}\left[\rho_{A}^{2}\right]\right)$ is almost minimal and then apply Markov's inequality. We have, for the expected purity of a subsystem,

$$
\begin{aligned}
\operatorname{Tr}\left[\rho_{A}^{2}\right] & =\mathbb{E}_{\psi \sim v}\left(\operatorname{Tr}\left[\rho_{A}^{2}\right]\right) \\
& =\mathbb{E}_{\psi \sim v}\left(\operatorname{Tr}\left[\mathbb{F}_{A, \bar{A}} \rho_{A}^{\otimes 2}\right]\right) \\
& =\operatorname{Tr}\left\{\mathbb{F}_{A, \bar{A}} \operatorname{Tr}_{B, \bar{B}}\left[\mathbb{E}(|\psi\rangle\langle\psi|)^{\otimes 2}\right]\right\} \\
& =\operatorname{Tr}\left[\mathbb{F}_{A, \bar{A}} \otimes \mathbb{1}_{B, \bar{B}} \mathbb{E}(|\psi\rangle\langle\psi|)^{\otimes 2}\right] .
\end{aligned}
$$

In the same graphical notation as in the proof of Lemma 3, this amounts to

$$
\mathbb{E} \operatorname{Tr}\left[\rho_{A}^{2}\right]=\sum_{\{\mathbb{1}, \mathbb{F}\}^{2 n}}
$$

here depicted for the subset $A$ corresponding to every $k=3$ spin. Similar to the strategy laid out in Ref. [26], we sum over every lower ball to obtain a statistical model. For this, we define the following two interactions. These are

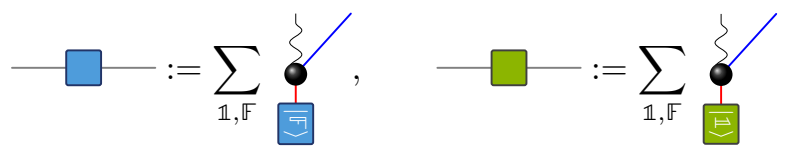

Graphically, this yields the chain

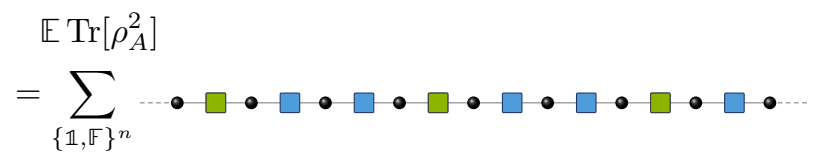

In order to proceed, we compute

$$
\begin{aligned}
& \mathbb{F} \square \mathbb{F}=1, \\
& \mathbb{1} \square \mathbb{1}=\frac{d D^{2}-d}{D^{2} d^{2}-1}=\eta(d, D), \\
& \mathbb{1} \square=0, \\
& \mathbb{E} \square \mathbb{E}=\frac{d^{2} D-D}{D^{2} d^{2}-1}=\eta(D, d)
\end{aligned}
$$

with the notation

$$
\eta(x, y):=\frac{x y^{2}-x}{x^{2} y^{2}-1}
$$

For the green plaquettes, we simply switch the roles of $\mathbb{1}$ and $\mathbb{F}$. As an intermediate step, let us compute the same but with $j$ blue plaquettes. The sum over the black balls is implicit, and the following is obtained by simply counting how many switches between $\mathbb{1}$ and $\mathbb{F}$ are allowed: 


$$
\begin{aligned}
& \mathbb{F} \square \cdot \square \cdot \square \mathbb{F}=1, \\
& \mathbb{1} \square \circ \square \circ \square \mathbb{1}=\eta(d, D)^{j}, \\
& \mathbb{1} \square \cdot \square \cdot \square \mathbb{F}=0 \text {, } \\
& \mathbb{F} \square \circ \square \circ \square \mathbb{1}=\eta(D, d) \sum_{l=1}^{j} \eta(d, D)^{j-l} \\
& =\eta(D, d) \frac{1-\eta(d, D)^{j}}{1-\eta(d, D)}
\end{aligned}
$$

We can group the chain in Eq. (36) into blocks of $k-1$ spins consisting of all spins to the right of a green plaquette except the one before the next green plaquette. For $k \geq$ 2 , we have, using Eq. (39) with $j=k-1$ combined with Eq. (37) for the final green plaquette,

$$
\begin{aligned}
& \mathbb{1} \square \cdot \square \cdot \square \cdot \square \mathbb{1} \\
& =\eta(d, D)^{k-1}+\eta(D, d)^{2} \frac{1-\eta(d, D)^{k-1}}{1-\eta(d, D)} \\
& \leq \eta(d, D)^{k-1}+\eta(D, d), \\
& \mathbb{1} \square \cdot \square \cdot \square \cdot \square \mathbb{F} \\
& =\eta(D, d) \\
& \leq \eta(d, D)^{k-1}+\eta(D, d), \\
& \mathbb{F} \square \cdot \square \cdot \square \cdot \square \mathbb{1} \\
& =\eta(d, D) \eta(D, d) \frac{1-\eta(d, D)^{k-1}}{1-\eta(d, D)} \\
& \leq \eta(D, d)+\eta(d, D)^{k-1}, \\
& \mathbb{F} \square \cdot \square \cdot \square \cdot \square \mathbb{F}=\eta(d, D) \text {. }
\end{aligned}
$$

In this step, we have used the facts that $\eta(x, y) \leq 1 / x$ and, for $x \geq 2$,

$$
\frac{1}{1-\eta(x, y)} \leq 2
$$

We sum over the number of spins with value 1 . We make use of the fact that every spin with value $\mathbb{1}$ contributes a factor of $\eta(d, D)^{k-1}+\eta(D, d)$ (via the plaquette to its right), to find that

$$
\begin{aligned}
\mathbb{E} \operatorname{Tr}\left[\rho_{A}^{2}\right] \leq & \sum_{i=0}^{n / k}\left(\begin{array}{c}
\frac{n}{k} \\
i
\end{array}\right)\left[\eta(d, D)^{k-1}+\eta(D, d)\right]^{i} \eta(d, D)^{n / k-i} \\
= & {\left[\eta(d, D)^{k-1}+\eta(D, d)+\eta(d, D)\right]^{n / k} } \\
= & \eta(d, D)^{n / k}\left[1+\eta(d, D)^{k-2}\right. \\
& \left.+\eta(D, d) \eta(d, D)^{-1}\right]^{n / k}
\end{aligned}
$$

Hence, for $d^{(1 / 2)} \leq D$ and large enough $k$, this expectation value becomes arbitrarily close to the minimal value $d^{-n / k}$. In the regime $D^{2} \leq d$, the Rényi-2 entropy is bounded by the entanglement area law [38]

$$
|\partial A| \log (D)=\frac{2 n}{k} \log (D) .
$$

As in the proof of Theorem 1, the expression for the expectation value in Eq. (42) implies a concentration result via Markov's inequality and the union bound. This concentration inequality extends to the Rényi-2 entropy.

\section{MAXIMUM ENTROPY FOR SMALL CONNECTED SUBSYSTEMS}

In this section, we show that small connected subsystems will, with high probability, feature a close to maximum entropy. A principle of maximum entropy for translation-invariant RMPSs has been proved in Refs. [11,17].

Theorem 3 (Almost maximum entropy for reduced states). Let $A$ be a subset of l consecutive qudits, and let $\rho_{A}^{\prime}$ be the normalized density matrix of a RMPS drawn from $\mu_{d, n, D}$ reduced to $A$. Then, for any real $r$,

$$
\operatorname{Pr}\left\{\operatorname{Tr}\left[\rho_{A}^{\prime 2}\right] \geq \Omega\left(D^{-r}\right)+d^{-l}\right\} \leq O\left(D^{-(2-r)}\right)
$$

for

$$
n \geq 2 \frac{\log D}{\log d}+l
$$

Proof. Let $\rho_{A}$ be the unnormalized quantum state. In the disordered case we consider here, we obtain analogously to the previous section the expression

$$
=\sum_{\{\mathbb{1}, \mathbb{F}\}^{n}} \mathbb{E} \operatorname{Tr}\left[\rho_{A}^{2}\right]
$$

for the subset $A$ consisting of $l$ consecutive qudits. Summing over the four contributions in Eq. (39), we obtain

$$
\begin{aligned}
\mathbb{E} \operatorname{Tr}\left[\rho_{A}^{2}\right]= & \eta(d, D)^{n-l}+\eta(d, D)^{l}+0 \\
& +\eta(D, d)^{2} \frac{1-\eta(D, d)^{l}}{1-\eta(D, d)} \frac{1-\eta(D, d)^{n-l}}{1-\eta(D, d)} .
\end{aligned}
$$

By Eq. (41), for $d, D \geq 2$,

$$
\mathbb{E} \operatorname{Tr}\left[\rho_{A}^{2}\right] \leq \frac{1}{d^{l}}+\frac{1}{d^{n-l}}+4 \frac{1}{D^{2}}
$$


holds. Let $N=\operatorname{Tr}[\rho]$ be the norm squared of the MPS. We have

$$
\operatorname{Tr}\left[\rho_{A}^{2}\right] \geq N^{2} \frac{1}{d^{l}},
$$

and using [see Eq. (67) in the proof of Lemma 1]

$$
\mathbb{E}\left(N^{2}\right)=1+\eta(d, D)^{n} \geq 1,
$$

we find that

$$
\begin{aligned}
\mathbb{E}\left(\operatorname{Tr}\left[\rho_{A}^{2}\right]-N^{2} d^{-l}\right) & \leq 4 \frac{1}{D^{2}}+\frac{1}{d^{n-l}}+\frac{1}{d^{n+l}} \\
& \leq 6 \frac{1}{D^{2}},
\end{aligned}
$$

where we have used

$$
n \geq 2 \frac{\log D}{\log d}+l, \quad \text { i.e., } d^{l-n} \leq \frac{1}{D^{2}} .
$$

Markov's inequality then yields

$$
\operatorname{Pr}\left(\operatorname{Tr}\left[\rho_{A}^{2}\right]-N^{2} d^{-l} \geq D^{-r}\right) \leq \frac{1}{6} D^{-(2-r)} .
$$

We now need to ensure that the normalization of the state does not worsen the bound. We employ the union bound in a similar manner as in the proof of Theorem 1. Let $\rho_{A}^{\prime}=$ $\rho_{A} / N$ be the normalized state. Suppose that

$$
\operatorname{Tr}\left[\rho_{A}^{2}\right]-N^{2} d^{-l} \leq D^{-r}
$$

and $|N-1| \leq \epsilon$. Then $N^{2} \geq(1-\epsilon)^{2}$ and

$$
\operatorname{Tr}\left[\rho_{A}^{\prime 2}\right]-d^{-l} \leq \frac{D^{-r}}{(1-\epsilon)^{2}} .
$$

Pick $\epsilon:=t d^{-k n}$, with $k \leq 1-r / 4$ and $t<1$. Then $\epsilon \leq$ $t d^{k l} D^{-2 k}$ and

$$
\operatorname{Tr}\left[\rho_{A}^{\prime 2}\right]-d^{-l} \leq O\left(D^{-r}\right) .
$$

By the same union bound argument as in the proof of Theorem 1, we get

$$
\begin{aligned}
\operatorname{Pr} & \left\{\operatorname{Tr}\left[\rho_{A}^{\prime}\right]-d^{-l} \leq O\left(D^{-r}\right)\right\} \\
& \geq \operatorname{Pr}\left\{\operatorname{Tr}\left[\rho_{A}\right]-N^{2} d^{-l} \leq O\left(D^{-r}\right)\right\}-\operatorname{Pr}\left(|N-1| \leq d^{-k n}\right) \\
& \geq 1-O\left(D^{-(2-r)}\right)-d^{-(1-2 k) n} \\
& \geq 1-O\left(D^{-(2-r)}\right),
\end{aligned}
$$

where we have used the fact that $k \leq 1-r / 4$ and hence $d^{(1-2 k) n} \geq D^{2-r}$. This concludes the proof.
The bound on the expectation value (47) is of the form

$$
\mathbb{E} \operatorname{Tr}\left[\rho_{A}^{2}\right] \leq d^{-l}+O\left(D^{-2}\right)+O\left(d^{l-n}\right) .
$$

In comparison, a bound for the expectation value of the form $d^{-l}+O\left(D^{-1 / 10}\right)$ for $D \geq n^{5}$ has been obtained in Ref. [17] for random translation-invariant MPSs. For periodic boundary conditions, a similar result has been proven in Ref. [11]. Perhaps not surprisingly, our bound in the disordered case scales slightly better in $D$. Moreover, the bond dimension $D$ is not required to grow with the system size $n$.

In Ref. [17], Levy's lemma has been employed in order to obtain an exponential concentration bound. This is not possible in our case, as the Lipschitz constant of the purity is necessarily lower bounded by $O(D)$. As a matter of fact, consider the MPS generated by choosing

$$
U^{(k)}:=\mathbb{1}_{d} \otimes \mathbb{1}_{D}
$$

for all $k$, i.e., $\rho=D^{2}|0\rangle\left\langle\left. 0\right|^{\otimes n}\right.$. Then, for any $A, \operatorname{Tr}\left[\rho_{A}^{2}\right]=$ $D^{2}$. Now pick a site $k$ and a unitary

$$
U:=\mathbb{1}_{d} \otimes V
$$

with $\operatorname{Tr}(V)=0$. For example, we can choose

$$
V:=\operatorname{diag}\left(e^{2 \pi \mathrm{i} j / D}\right)_{j=0, \ldots, D-1} .
$$

We construct the MPS $\sigma$ with the identity on every site and $U$ on site $k$. For any $A$ containing $k, \operatorname{Tr}\left[\sigma_{A}^{2}\right]=0$. The Lipschitz constant of the function

$$
\begin{aligned}
& U(d D)^{\times n} \rightarrow \mathbb{R}, \\
& (U) \mapsto \operatorname{Tr}\left[\rho_{A}^{U^{2}}\right],
\end{aligned}
$$

where $\rho^{U}$ is the MPS constructed with the unitaries and where we equip $U(d D)^{\times n}$ with the product Frobenius norm, is then bounded by

$$
L \geq \frac{\left|\operatorname{Tr}\left[\rho_{A}^{2}\right]-\operatorname{Tr}\left[\sigma_{A}^{2}\right]\right|}{\|\mathbb{1}-U\|_{2}} \geq \frac{D^{2}}{2 D}=O(D),
$$

where we have made use of the triangle inequality to further bound the denominator.

\section{CONCENTRATION AROUND THE UNIT NORM}

In this section, we make use of the machinery of statistical mechanics mappings in order to show that the norm of the vectors $|\psi\rangle$ is exponentially concentrated around unity, i.e., around being an actual quantum state.

Lemma 6 (Concentration around a unit norm). It holds that

$$
\operatorname{Pr}(|\langle\psi \mid \psi\rangle-1| \geq \varepsilon) \leq \varepsilon^{-2} d^{-n}
$$


To prove this statement, first note that

$$
\mathbb{E}\langle\psi \mid \psi\rangle^{2}=\operatorname{Tr}\left[\mathbb{F}(|\psi\rangle\langle\psi|)^{\otimes 2}\right] .
$$

Moreover, using first-order Weingarten calculus, it is easy to see that $\mathbb{E}\langle\psi \mid \psi\rangle=1$. Graphically, this corresponds to the spin chain depicted in Eq. (34) with only blue plaquettes as

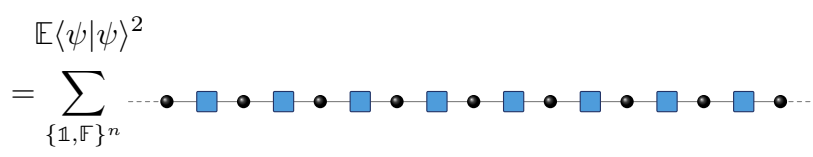

However, given the values of the plaquettes in Eq. (37), the only nonzero contributions are all spins $\mathbb{1}$ or all spins $\mathbb{F}$. This yields

$$
\mathbb{E}\langle\psi \mid \psi\rangle^{2}=1+\left(\frac{d D^{2}-d}{D^{2} d^{2}-1}\right)^{n} \leq 1+d^{-n} .
$$

Now from Markov's inequality we obtain

$$
\operatorname{Pr}\left[(\langle\psi \mid \psi\rangle-1)^{2} \geq \varepsilon\right] \leq \varepsilon^{-1} \mathbb{E}(1-\langle\psi \mid \psi\rangle)^{2} \leq \varepsilon^{-1} d^{-n}
$$

This exponential concentration allows us to prove all other concentration results without regarding the normalization. In fact, we combine every calculation with a union bound and the above concentration result such that the normalization does not change the statements. It is also noteworthy that all these concentration results follow from simple applications of Markov's inequality and do not require geometric methods such as Levy's lemma [45].

\section{LOCAL EXPECTATION VALUES}

Consider an observable $O$ acting on $\left(\mathbb{C}^{d}\right)^{\otimes l}$ for some small number of sites $l$. Here we consider the expectation values $\left\langle\psi\left|O \otimes \mathbb{1}_{\left(\mathbb{C}^{2}\right) \otimes n-l}\right| \psi\right\rangle$. We assume without loss of generality that $O$ is traceless. First, note that we have

$$
\mathbb{E}\left\langle\psi\left|O \otimes \mathbb{1}_{\left(\mathbb{C}^{2}\right)^{\otimes n-l}}\right| \psi\right\rangle=\operatorname{Tr}(O)=0 .
$$

We want to show that the expectation values are concentrated around 0 . One possible way to do this is to exploit the results of Sec. IV to argue that the local density matrix is close in norm with high probability to the maximally mixed state, as done in Ref. [17], and conclude by Hölder's inequality that, for the expectation value expressed in terms of the reduced density matrix $\rho$, the following holds:

$$
|\operatorname{Tr}[O \rho]|=\left|\operatorname{Tr}\left[O\left(\rho-\mathbb{1} / d^{l}\right)\right]\right| \leq\left\|\rho-\mathbb{1} / d^{l}\right\|_{\infty}\|O\|_{1} .
$$

Here $\|O\|_{1}$ is a constant in $n$ and $D$. Nevertheless, we want to showcase how our method can be applied directly to this problem.

To showcase the flexibility of our approach, we provide a direct bound on the second moment

$\mathbb{E}\left\langle\psi\left|O \otimes \mathbb{1}_{\left(\mathbb{C}^{2}\right) \otimes n-l}\right| \psi\right\rangle^{2}=\mathbb{E} \operatorname{Tr}\left[\left(O \otimes \mathbb{1}_{\left(\mathbb{C}^{2}\right) \otimes n-l}|\psi\rangle\langle\psi|\right)^{\otimes 2}\right]$.

We consider the case $l=1$ for simplicity. Graphically, we have

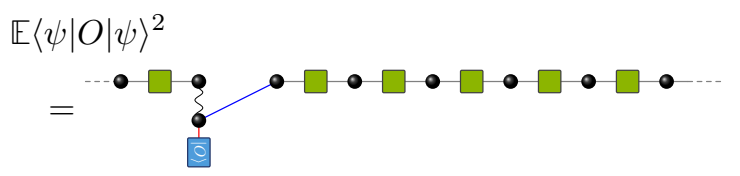

where $|O\rangle:=[(\mathbb{1} \otimes O)|\Omega\rangle]^{\otimes 2}$. We obtain the interactions

$$
\begin{aligned}
& \mathbb{1} \underset{0}{1}=-\frac{\operatorname{Tr}\left[O^{2}\right]}{D^{2} d^{3}-d}, \\
& \mathbb{F} \mathfrak{q}=\frac{\operatorname{Tr}\left[O^{2}\right] D^{2}}{D^{2} d^{2}-1} \text {, } \\
& \mathbb{1} \underset{0}{-1} \mathbb{F}=-\frac{\operatorname{Tr}\left[O^{2}\right] D}{D^{2} d^{3}-d} \text {, } \\
& \mathbb{F} \mathfrak{1}=\frac{\operatorname{Tr}\left[O^{2}\right] D}{D^{2} d^{2}-1}
\end{aligned}
$$

from this. Simply by ignoring the one negative contribution (all spins $\mathbb{1}$ ), we obtain

$$
\begin{aligned}
& \mathbb{E}\left\langle\psi\left|O \otimes \mathbb{1}_{\left(\mathbb{C}^{2}\right) \otimes n-l}\right| \psi\right\rangle^{2} \\
& \leq \operatorname{Tr}\left[O^{2}\right]\left[\frac{D}{D^{2} d^{2}-1} \eta(D, d) \sum_{i=0}^{n-2} \eta(d, D)^{i}\right. \\
&\left.+\frac{D^{2}}{D^{2} d^{2}-1}\left(\frac{d^{2} D-D}{D^{2} d^{2}-D}\right)^{n-2}\right] \\
& \leq \operatorname{Tr}\left[O^{2}\right]\left(D^{-2} \frac{1-d^{-n+1}}{1-d^{-1}}+D^{-n+1}\right) \\
& \leq 2 D^{-2} \operatorname{Tr}\left[O^{2}\right]
\end{aligned}
$$

for $n \geq 2$ as a combinatorial bound. We can now achieve a concentration result for local expectation values from Markov's inequality. Previously, typicality of expectation values was argued for translation-invariant MPSs in 
Ref. [46], where the authors argued an exponential concentration if $D$ grows faster than $\Omega\left(n^{2}\right)$. However, their proof is based on the concentration of measure phenomenon and the bound they obtained on the Lipshitz constant involves the assumption that the second highest eigenvalue of the transfer operator is small enough for essentially all instances. While a numerical assessment indicates that this is the case for all practical purposes, it is difficult to obtain explicit rigorous bounds for the dependence on $n$ this way. Our simple bound from second moments only scales as $D^{-2}$ (as opposed to $e^{-\Omega(D)}$ ), but it is independent of $n$ and does not require any further assumptions.

\section{OUTLOOK}

In this work, we have systematically explored RMPSs where the individual tensors have been chosen independently according to the Haar measure. Exploiting a mapping to a one-dimensional statistical mechanics model, we have been in the position to compute expectation values of various quantities for such disordered RMPSs. We have derived concentration results for the effective dimension, implying equilibration under the time evolution of generic Hamiltonians, and concentration results for the Rényi-2 entropy and the expectation values of local observables.

While we have put properties of such families of quantum states into the focus of our analysis, it should be clear that by means of the parent Hamiltonian concept mentioned above, we could have discussed ensembles of local Hamiltonians. For translational-invariant RMPSs [12], this picture is particularly transparent.

An obvious further problem is to consider quantities that require higher moments, such as higher Rényi entropies $S_{\alpha}$ with $2 \leq \alpha \in \mathbb{Z}$. This would result in more complicated statistical models with $\alpha$ ! many local degrees of freedom. Another open question concerns higher-dimensional systems. It would be interesting to apply a similar analysis to projected entangled pair states.

For translational-invariant RMPSs, the expected correlation length has been proven in Refs. [11]. The family of states defined in this fashion gives rise to generic representatives of the trivial phase of matter [9] with unit probability. If a state has symmetries, i.e., if the state vector satisfies $U_{g}^{\otimes n}|\psi\rangle=e^{i \theta_{g}}|\psi\rangle$ for some real phase $\theta_{g}$, and where $g \mapsto U_{g}$ is a linear unitary representation of a symmetry group $G$, then this symmetry is (for a suitable phase gauge) reflected on the virtual level of the MPS as a projective unitary representation of group $G$, satisfying

$$
V_{g} V_{h}=e^{i \omega(g, h)} V_{g h}
$$

for a real phase $(g, h) \mapsto \omega(g, h)$ [47]. Different phases of matter respecting these symmetries in symmetry-protected topological order are now captured by equivalence classes, called cohomology classes, they again forming a group, the second cohomology group $H_{2}[G, U(1)]$ of $G$ over $U(1)$ $[9,48,49]$. That said, it now makes sense to think of RMPSs that respect a physical symmetry and think of typical symmetry-protected topological (SPT) phases of matter. Here, the Haar measure is chosen in each of the blocks of a direct sum on the virtual level, respecting the projective unitary representation of group $G$. In this sense, one can speak of common representatives of SPT phases, a line of thought that will be elaborated upon elsewhere.

More broadly put, this work can be seen as a contribution to a bigger program concerned with understanding generic phases of quantum matter by means of random tensor networks. Indeed, properties of random tensor networks can often be easier computed than those of tensor networks in which the entries are specifically chosen. Randomness hence serves as a computational tool, a line of thought that can be dated back to Ref. [3] and further.

In Ref. [50], such a line of thought has already been applied to identify properties of holographic tensor networks, where a desirable property to be a so-called perfect tensor turns out to the approximately satisfied with high probability. Building upon this insight, one can compute properties of the resulting boundary state. More ambitiously still, it makes a lot of sense to think of holographic random tensor network models in which the tensors have further structure, e.g., to be match-gate tensor networks [51]. Similarly, questions of properties of higherdimensional cubic tensor networks arise along similar lines. It is the hope that the present work can provide insights and a powerful machinery to address such further questions when exploring typical instances of phases of matter.

\section{ACKNOWLEDGMENTS}

We would like to warmly thank Alexander Altland, Alex Goeßmann, Dominik Hangleiter, Nick Hunter-Jones, Richard Kueng, Amin Thainat, and Carolin Wille for fruitful discussions. We would like to thank the DFG (CRC 183, project A03, FOR 2724, EI 519/15-1, EI 519/17-1) and the FQXi for support.

\section{APPENDIX}

A natural follow up question is whether RMPSs have features similar to generic Haar-random states. Naively, an argument as above might be used to show that RMPSs form approximate spherical 2-designs [31]. The difference of moment operators in the 2-norm can be bounded using the frame potential as

$$
\left\|\mathbb{E}_{\psi \sim v}(|\psi\rangle\langle\psi|)^{\otimes 2}-\frac{2 P_{\text {sym }}}{d^{n}\left(d^{n}+1\right)}\right\|_{F}^{2}=\mathcal{F}_{2, \nu}-\frac{2}{d^{n}\left(d^{n}-1\right)},
$$


and the frame potential is given by

$$
\mathcal{F}_{2, \nu}:=\mathbb{E}_{\psi, \phi \sim v}|\langle\psi \mid \phi\rangle|^{4},
$$

which is reminiscent of the expression in Eq. (11). After all, random product states constitute an exact projective 1design.

However, RMPSs with polynomially bounded bond dimensions have low-entanglement structure by definition. Since the Schmidt rank along any bipartition is bounded by $D$, the purity is bounded below by $1 / D$, but the average over this quantity is exponentially small for an approximate 2-design. In more detail, consider a probability measure such that

$$
\left\|\mathbb{E}_{\psi \sim v}(|\psi\rangle\langle\psi|)^{\otimes 2}-\frac{2 P_{\text {sym }}}{d^{n}\left(d^{n}+1\right)}\right\|_{F} \leq \varepsilon .
$$

With this notion we obtain a straightforward bound on the entanglement purity over a bipartition of the spin chain into subsets $A$ and $B$ of equal size $n / 2$. With the norm inequality $\|\cdot\|_{1} \leq \sqrt{\operatorname{dim} \mathcal{H}}\|\cdot\|_{F}$ [see, e.g., Eq. (1.2.6) of Ref. [52] ], we obtain

$$
\begin{aligned}
\mathbb{E}_{\psi \sim v}\left(\operatorname{Tr}\left[\rho_{A}^{2}\right]\right) & =\mathbb{E}_{\psi \sim v}\left(\operatorname{Tr}\left[\mathbb{F}_{A, \bar{A}} \rho_{A}^{\otimes 2}\right]\right) \\
& =\operatorname{Tr}\left\{\mathbb{F}_{A, \bar{A}} \operatorname{Tr}_{B, \bar{B}}\left[\mathbb{E}_{\psi \sim v}(|\psi\rangle\langle\psi|)^{\otimes 2}\right]\right\}
\end{aligned}
$$

Therefore,

$$
\begin{aligned}
& \mathbb{E}_{\psi \sim v}\left(\operatorname{Tr}\left[\rho_{A}^{2}\right]\right) \\
& \quad \leq \frac{2}{d^{n}\left(d^{n}+1\right)} \operatorname{Tr}\left\{\mathbb{F}_{A, \bar{A}} \operatorname{Tr}_{B, \bar{B}}\left[P_{\mathrm{sym}}\right]\right\}+d^{n} \varepsilon \\
& =\frac{1}{d^{n}\left(d^{n}+1\right)} \operatorname{Tr}\left\{\mathbb{F}_{A, \bar{A}} \operatorname{Tr}_{B, \bar{B}}\left[\mathbb{F}_{A B, \overline{A B}}+\mathbb{1}_{A B, \overline{A B}}\right]\right\}+d^{n} \varepsilon \\
& =\frac{2 d^{3 n / 2}}{d^{n}\left(d^{n}+1\right)}+d^{n} \varepsilon .
\end{aligned}
$$

As a small expectation value implies the existence of instances with small values, this leads to a contradiction for

$$
\varepsilon \leq \frac{d^{-n}}{D}
$$

This argument rules out a "cutoff" phenomenon, where the error is exponentially suppressed in the bond dimension $D$ only after some polynomial threshold has been surpassed.

[1] G. Akemann, J. Baik, P. Di Francesco, editors., The Oxford Handbook of Random Matrix Theory (Oxford University Press, Oxford, 2015).
[2] P. Hayden, D. Leung, P. W. Shor, and A. Winter, Randomizing quantum states: Constructions and applications, Comm. Math. Phys. 250, 371 (2004).

[3] P. Hayden, D. W. Leung, and A. Winter, Aspects of generic entanglement, Comm. Math. Phys. 265, 95 (2006).

[4] J. Sánchez-Ruiz, Simple proof of page's conjecture on the average entropy of a subsystem, Phys. Rev. E 52, 5653 (1995).

[5] D. Gross, S. T. Flammia, and J. Eisert, Most Quantum States are too Entangled to be Useful as Computational Resources, Phys. Rev. Lett. 102, 190501 (2009).

[6] J. Eisert, M. Friesdorf, and C. Gogolin, Quantum manybody systems out of equilibrium, Nat. Phys. 11, 124 (2015).

[7] C. Gogolin and J. Eisert, Equilibration, thermalisation, and the emergence of statistical mechanics in closed quantum systems, Rep. Prog. Phys. 79, 56001 (2016).

[8] A. Polkovnikov, K. Sengupta, A. Silva, and M. Vengalattore, Non-equilibrium dynamics of closed interacting quantum systems, Rev. Mod. Phys. 83, 863 (2011).

[9] N. Schuch, D. Perez-Garcia, and I. Cirac, Classifying quantum phases using matrix product states and projected entangled pair states, Phys. Rev. B 84, 165139 (2011).

[10] S. Garnerone, T. R. de Oliveira, S. Haas, and P. Zanardi, Statistical properties of random matrix product states, Phys. Rev. A 82, 052312 (2010).

[11] C. E. Gonzales-Guillen, M. Junge, and I. Nechita, On the spectral gap of random quantum channels. arXiv:1811.08847.

[12] C. Lancien and D. Pérez-García, Correlation length in random MPS and PEPS. arXiv:1906.11682.

[13] D. A. Abanin, E. Altman, I. Bloch, and M. Serbyn, Colloquium: Many-body localization, thermalization, and entanglement, Rev. Mod. Phys. 91, 021001 (2019).

[14] M. Friesdorf, A. H. Werner, W. Brown, V. B. Scholz, and J. Eisert, Many-Body Localisation Implies That Eigenvectors are Matrix-Product States, Phys. Rev. Lett. 114, 170505 (2015).

[15] D. A. Huse, R. Nandkishore, and V. Oganesyan, Phenomenology of fully many-body-localized systems, Phys. Rev. B 90, 174202 (2014).

[16] A. Rolandi and H. Wilming, Extensive Rényi entropies in matrix product states. arXiv:2008.11764, (2020).

[17] B. Collins, C. E. González-Guillén, and D. Pérez-García, Matrix product states, random matrix theory and the principle of maximum entropy. arXiv:1201.6324, (2012).

[18] R. Movassagh and J. Schenker, An ergodic theorem for homogeneously distributed quantum channels with applications to matrix product states. arXiv:1909.11769, (2019).

[19] R. Movassagh and J. Schenker, Theory of ergodic quantum processes, arXiv:2004.14397, (2020).

[20] F. Arute et al., Quantum supremacy using a programmable superconducting processor, Nature 574, 505 (2019).

[21] C. Neill, P. Roushan, K. Kechedzhi, S. Boixo, S. V. Isakov, V. Smelyanskiy, R. Barends, B. Burkett, Y. Chen, and Z. Chen, A blueprint for demonstrating quantum supremacy with superconducting qubits, Science 360, 195 (2017).

[22] F. G. S. L. Brandão, A. W. Harrow, and M. Horodecki, Local random quantum circuits are approximate polynomial-designs, Commun. Math. Phys. 346, 397 (2016). 
[23] F. G. S. L. Brandao, W. Chemissany, N. Hunter-Jones, R. Kueng, and J. Preskill, Models of quantum complexity growth. arXiv:1912.04297.

[24] A. Chandran and C. R. Laumann, Semiclassical limit for the many-body localization transition, Phys. Rev. B 92, 024301 (2015).

[25] J. Haferkamp, F. Montealegre-Mora, M. Heinrich, J. Eisert, D. Gross, and I. Roth, Quantum homeopathy works: Efficient unitary designs with a system-size independent number of non-clifford gates. arXiv:2002.09524.

[26] N. Hunter-Jones, Unitary designs from statistical mechanics in random quantum circuits. arXiv:1905.12053, (2019).

[27] A. Nahum, S. Vijay, and J. Haah, Operator Spreading in Random Unitary Circuits, Phys. Rev. X 8, 021014 (2018).

[28] C. Sünderhauf, D. Pérez-García, D. A. Huse, N. Schuch, and J. I. Cirac, Localization with random time-periodic quantum circuits, Phys. Rev. B 98, 134204 (2018).

[29] T. Zhou and A. Nahum, Emergent statistical mechanics of entanglement in random unitary circuits, Phys. Rev. B 99, 174205 (2019).

[30] M. Kliesch, R. Kueng, J. Eisert, and D. Gross, Guaranteed recovery of quantum processes from few measurements, Quantum 3, 171 (2019).

[31] D. Gross, K. Audenaert, and J. Eisert, Evenly distributed unitaries: On the structure of unitary designs, J. Math. Phys. 48, 052104 (2007).

[32] M. Fannes, B. Nachtergaele, and R. F. Werner, Finitely correlated states on quantum spin chains, Commun. Math. Phys. 144, 443 (1992).

[33] D. Perez-Garcia, F. Verstraete, M. M. Wolf, and J. I. Cirac, Matrix product state representations, Quantum Inf. Comput. 7, 401 (2007).

[34] D. Gross and J. Eisert, Quantum computational webs, Phys. Rev. A 82, 040303(R) (2010).

[35] D. Perez-Garcia, F. Verstraete, M. M. Wolf, and J. I. Cirac, Matrix product state representations, Quant. Inf. Comp. 5 and 6, 401 (2007).

[36] N. Linden, S. Popescu, A. J. Short, and A. Winter, Quantum mechanical evolution towards thermal equilibrium, Phys. Rev. E 79, 061103 (Jun 2009).

[37] B. Bauer and C. Nayak, Area laws in a many-body localised state and its implications for topological order, J. Stat. Mech. 2013, P09005 (2013).
[38] J. Eisert, M. Cramer, and M. B. Plenio, Area laws for the entanglement entropy, Rev. Mod. Phys. 82, 277 (2010).

[39] Y. Huang and A. W. Harrow, Instability of localization in translation-invariant systems, arXiv:1907.13392 (2019).

[40] P. Reimann, Foundation of Statistical Mechanics under Experimentally Realistic Conditions, Phys. Rev. Lett. 101, 190403 (2008).

[41] H. Tasaki, From Quantum Dynamics to the Canonical Distribution: General Picture and a Rigorous Example, Phys. Rev. Lett. 80, 1373 (1998).

[42] P. W. Brouwer and C. W. J. Beenakker, Diagrammatic method of integration over the unitary group, with applications to quantum transport in mesoscopic systems, J. Math. Phys. 37, 4904 (1996).

[43] B. Collins and P. Śniady, Integration with respect to the haar measure on unitary, orthogonal and symplectic group, Comm. Math. Phys. 264, 773 (2006).

[44] H. Wilming, M. Goihl, I. Roth, and J. Eisert, EntanglementErgodic Quantum Systems Equilibrate Exponentially Well, Phys. Rev. Lett. 123, 200604 (2019).

[45] M. Ledoux, The concentration of measure phenomenon. Number 89. American Mathematical Soc., (2001).

[46] S. Garnerone, T. R. de Oliveira, and P. Zanardi, Typicality in random matrix product states, Phys. Rev. A 81, 032336 (2010).

[47] M. Sanz, M. M. Wolf, D. Pérez-García, and J. I. Cirac, Matrix product states: Symmetries and two-body hamiltonians, Phys. Rev. A 79, 042308 (2009).

[48] X. Chen, Z.-C. Gu, and X.-G. Wen, Classification of gapped symmetric phases in one-dimensional spin systems, Phys. Rev. B 83, 035107 (2011).

[49] F. Pollmann, A. M. Turner, E. Berg, and M. Oshikawa, Entanglement spectrum of a topological phase in one dimension, Phys. Rev. B 81, 064439 (2010).

[50] P. Hayden, S. Nezami, X.-L. Qi, N. Thomas, M. Walter, and Z. Yang, Holographic duality from random tensor networks, J. High En. Phys. 2016, 9 (2016).

[51] A. Jahn, M. Gluza, F. Pastawski, and J. Eisert, Holography and criticality in matchgate tensor networks, Sci. Adv. 5, eaaw0092 (2019).

[52] R. A. Low, Pseudo-randomness and learning in quantum computation. arXiv:1006.5227, (2010). 